\title{
Simulation of anticoagulation in atrial fibrillation patients with rivaroxaban - from trial to target population
}

\author{
Chi Zhang ${ }^{1}$, Weiwei Wang ${ }^{2}$, Mangmang Pan $^{2}$, Hongfang $\mathrm{Shi}^{2}$, Zheng $\mathrm{Li}^{2}$, Long Shen ${ }^{1}$, and \\ Zhichun $\mathrm{Gu}^{1}$ \\ ${ }^{1}$ Shanghai Jiao Tong University School of Medicine Affiliated Renji Hospital \\ ${ }^{2}$ Affiliation not available
}

August 12, 2020

\begin{abstract}
Aim The population included in randomized controlled clinical trial of ROCKET AF and observational studies were different, and the effectiveness and safety of rivaroxaban in stroke prevention of atrial fibrillation (AF) varied among studies. The aim of this study was to estimate the real-world outcomes of rivaroxaban in AF patients in a relatively accurate way. Methods A discrete event simulation (DES) model was proposed to predict the counterfactual outcomes of ROCKET AF that would have it been conducted in broader observational study populations. The hypothetical cohorts of patients were generated using Monte Carlo simulation. The DES model structure was built based on disease progression and possible outcomes of AF. Cardiovascular events were recorded during the simulated two-year follow-up period. Results The results showed lower predicted rates of stroke/ systemic embolism (SE) and major bleeding in three observational studies than those in simulated ROCKET AF. The simulated stroke/SE incidence was 1.097-1.318 per 100 patient-years and simulated major bleeding incidence was 2.804-3.238 per 100 patient-years in observational studies. The risk difference of stroke/SE and major bleeding was similar among predicted outcomes of the three observational studies. Most simulated hazard ratios (HRs) were close to the corresponding observed HRs. Conclusion The simulated incidence of stroke/SE incidence and major bleeding might reflect the real-world event rate in AF patients. Even some differences existed in the absolute rates of stroke/SE and major bleeding between observed and simulated studies, the results confirmed similar effectiveness and safety to ROCKET AF comparing rivaroxaban and warfarin in $\mathrm{AF}$ patients.
\end{abstract}

Title:Simulation of anticoagulation in atrial fibrillation patients with rivaroxaban - from trial to target population

Running title: Simulation of rivaroxaban in AF patients

Authors: Chi Zhang, MD ${ }^{1 a}$; Wei-Wei Wang, PhD ${ }^{2 a}$; Mang-Mang Pan, $\mathrm{MD}^{1}$; Fang-Hong Shi, MD ${ }^{1}$; Zheng $\mathrm{Li}, \mathrm{MD}^{3}$; Shen Long, $\mathrm{MD}^{3}$; Zhi-Chun Gu, $\mathrm{MD}^{1^{*}}$

Affiliation of Authors: ${ }^{1}$ Department of Pharmacy, Renji Hospital, School of Medicine, Shanghai Jiao Tong University, Shanghai 200127, China; ${ }^{2}$ Department of Cloud Foundation, SAP (China) Co., Ltd., Shanghai 201203, China; ${ }^{3}$ Department of Cardiology, Renji Hospital, School of Medicine, Shanghai Jiao Tong University, Shanghai 200127, China.

${ }^{\text {a }}$ Chi Zhang and Wei-Wei Wang contributed equally to this work, and should be considered as co-first authors

\section{*Correspondence Author:}

Zhi-Chun Gu, MD, Department of Pharmacy, Renji Hospital, School of Medicine, Shanghai Jiao Tong University, Shanghai 200127, China. E-mail: guzhichun213@163.com 


\section{Abstract}

Aim

The population included in randomized controlled clinical trial of ROCKET AF and observational studies were different, and the effectiveness and safety of rivaroxaban in stroke prevention of atrial fibrillation (AF) varied among studies. The aim of this study was to estimate the real-world outcomes of rivaroxaban in AF patients in a relatively accurate way.

Methods

A discrete event simulation (DES) model was proposed to predict the counterfactual outcomes of ROCKET AF that would have it been conducted in broader observational study populations. The hypothetical cohorts of patients were generated using Monte Carlo simulation. The DES model structure was built based on disease progression and possible outcomes of AF. Cardiovascular events were recorded during the simulated two-year follow-up period.

Results

The results showed lower predicted rates of stroke/ systemic embolism (SE) and major bleeding in three observational studies than those in simulated ROCKET AF. The simulated stroke/SE incidence was 1.0971.318 per 100 patient-years and simulated major bleeding incidence was 2.804-3.238 per 100 patient-years in observational studies. The risk difference of stroke/SE and major bleeding was similar among predicted outcomes of the three observational studies. Most simulated hazard ratios (HRs) were close to the corresponding observed HRs.

Conclusion

The simulated incidence of stroke/SE incidence and major bleeding might reflect the real-world event rate in $\mathrm{AF}$ patients. Even some differences existed in the absolute rates of stroke/SE and major bleeding between observed and simulated studies, the results confirmed similar effectiveness and safety to ROCKET AF comparing rivaroxaban and warfarin in $\mathrm{AF}$ patients.

Keywords :rivaroxaban; atrial fibrillation $(\mathrm{AF})$; simulation; randomized controlled trial (RCT); observational study

\section{What is already known about this subject}

* The effectiveness and safety of rivaroxaban in stroke prevention of AF patients varied among studies (RCTs and observational studies).

* Difference in patient characteristics could be a common barrier leading to the outcome discrepancies across studies. Besides, difference in data source, outcome measures, and patient adherence, as well as confounding bias of observational studies, all contribute to the discrepant results.

\section{What this study adds}

* A DES model was proposed to predict the counterfactual outcomes of ROCKET AF that would have it been conducted in broader observational study populations.

* Both predicted stroke/SE rate and major bleeding rate were lower in the three observational studies than those in simulated ROCKET AF, which might reflect the real-world event rate in AF patients.

* The results confirmed similar effectiveness and safety to ROCKET AF comparing rivaroxaban and warfarin in the anticoagulation of AF patients.

Introduction

Atrial fibrillation (AF) is the most commonly diagnosed and treated arrhythmia in clinical practice with substantial and increasing health burden. In the United States, 2.7 to 6.1 million individuals are currently 
suffered, and its prevalence is estimated to be more than 8 million people by the year $2050^{1,2}$. Stroke is the most feared complication of AF, and oral anticoagulation could help prevent most cases of stroke ${ }^{3}$. Nonvitamin $\mathrm{K}$ antagonist oral anticoagulants (NOACs) now have become an alternative to vitamin $\mathrm{K}$ antagonists for preventing stroke in AF patients ${ }^{4}$. Rivaroxaban, one of the most used NOACs, was approved for stroke prevention in AF patients based on the pivotal randomized controlled clinical trial (RCT) of ROCKET AF ${ }^{5}$, which demonstrated the efficacy of rivaroxaban in reducing AF-related stroke risk. RCTs are conducted on highly selective populations and are managed in tightly controlled settings. Therefore, RCTs are considered as the gold standard for assessing treatment efficacy and the results are of highest reliability. Nevertheless, RCTs, such as ROCKET AF, usually exclude certain patient groups, for example, AF patients with $\mathrm{CHADS}_{2}$ score of 0 to $1^{5}$, which account for about $40 \%$ of the whole AF population in the real-world setting ${ }^{6,7}$. It is well known that AF patients with different stroke risk estimated by $\mathrm{CHADS}_{2}$ score might receive different benefits from anticoagulation therapy ${ }^{8}$. Such rigorous exclusions could inevitably limit the generalizability of evidence from trials, failing to be reflective of treatment benefits and risks in all patient populations actually treated in the real practice. Therefore, uncertainty exists when physicians making anticoagulation decisions of rivaroxaban for patients who have been excluded from ROCKET AF.

Non-RCTs using routine electronic healthcare databases, such as insurance claims data or registry data, are available for large and diverse patient populations, which could complete the RCTs by capturing rare adverse events and long-term outcomes, as well as provide outcome estimates of treatment effectiveness in broad patient populations. However, the results of observational studies often differ from those of RCTs, which might mainly result from the differences in patient characteristics, drug adherence, and outcome measurement across studies that differ in design ${ }^{9}$. XANTUS, a prospective, single-arm, observational study described the use of rivaroxaban in a broad unselected AF patient population ${ }^{6}$. ROCKET AF and XANTUS exhibited different baseline characteristics. Lower value of $\mathrm{CHADS}_{2}$ score was observed in patients of XANTUS, and the proportion of patients with prior stroke, heart failure, hypertension, or diabetes mellitus was also lower in XANTUS than that in ROCKET AF, which might lead to the outcome discrepancy between these two studies ${ }^{10}$.

Moreover, effectiveness and safety of rivaroxaban in AF patients are also varied among different observational studies. The incidence of stroke/systemic embolism (SE) was observed to be 0.8 event per 100 patient-years in XANTUS ${ }^{6}$, while different rates were detected as 1.9 and 4.6 per 100 patient-years in other two observational studies $^{7,11}$, respectively (Table S1). Such significant variations within observational studies might result from the difference in study design, data source, definition of outcomes, length of observation, analysis methods, et al. ${ }^{12}$. Accordingly, although real-world studies could support and extend RCT findings to large patient populations, the results could be suspect.

In order to obtain sustained results of rivaroxaban in the use of anticoagulation for AF patients, it is desirable to make the results of ROCKET AF and the real-world studies to mutually complete each other. To our knowledge, generalizing the baseline characteristics of RCT population to match those of real-world patients could facilitate generation of evidence for effectiveness and safety of treatments in excluded populations, thus providing more relevant evidence for decision-makers ${ }^{9}$. In this study, we propose a discrete event simulation (DES) to predict the counterfactual outcomes of ROCKET AF that would have it been conducted in broader observational study populations.

Methods

2.1 Data sources and study population

ROCKTE AF was selected as a case of RCT evaluating the efficacy and safety of rivaroxaban versus warfarin in AF patients. XANTUS, a prospective observational study, was chosen to investigate whether the results of rivaroxaban obtained in ROCKET AF could translate into real-world clinical practice. Two other observational studies (Laliberte 2014 and Amin 2017) were used to assess the effectiveness and safety of rivaroxaban versus warfarin in routine care. Patient baseline characteristics of the four studies were collected, including age, gender, previous thromboembolic events (stroke, systemic embolism-SE), transient 
ischaemic attack-TIA, and myocardial infarction-MI), heart failure (HF), hypertension, diabetes mellitus $(\mathrm{DM}), \mathrm{CHADS}_{2}$ risk of stroke, et al. The incidence rate of events, such as stroke/SE, major bleeding, intracranial hemorrhage ( $\mathrm{ICH}$ ), gastrointestinal (GI) bleeding, and MI, were extracted as events per 100 patient-years.

\subsection{Discrete event simulation model}

A discrete event simulation (DES) model was developed and reprogrammed in Python (version 3.7). The hypothetical cohorts of patients were generated according to the baseline covariate distributions that matched the marginal distribution of covariates reported in ROCKET AF, XANTUS, and the two observational studies (Laliberte 2014 and Amin 2017), respectively, using Monte Carlo simulation by randomly sampling. The random sampling continued until 7000 patients were simulated for each treatment group, which was similar to the sample size of ROCKET AF. Figure 1 presents the DES model structure built based on a priori knowledge about disease progression and possible outcomes of AF patients receiving rivaroxaban ${ }^{3,13}$. The model was designed to predict treatment outcomes conditional on patients' baseline characteristics. $\mathrm{CHADS}_{2}$ score of individual patients was calculated according to the simulated patient's baseline characteristics. Therefore, patients at different stroke risk would trace different probabilistic pathways in the model based on their treatment assignment (rivaroxaban or warfarin). The incidence of the events in the simulation model was obtained based on the rates reported in ROCKET AF (Table S2) ${ }^{5}, 14-18$. Cardiovascular events, such as stroke/SE, major bleeding (GI bleeding, ICH and other major bleeding), nonmajor clinically relevant bleeding (NMCR bleeding), MI, and unknown death, were recorded during the simulated two-year follow-up period. The patient's cardiovascular profile was updated from the first year to the second year. Patients who suffered death, fatal stroke/SE, fatal major bleeding, or fatal MI, were supposed to exit the simulation model after their outcomes were recorded. The event rates, hazard ratios (HRs), and risk differences (RDs) were calculated and reported.

\subsection{Comparison of simulated results to observed results in ROCKET AF}

To validate the DES model, we compare the simulated results and observed results of ROCKET AF. RDs and relative HRs (RHRs) of each outcome were calculated. RDs were estimated as the difference in the absolute risk of each outcome, and were calculated by subtracting the observed incidence from simulated incidence ${ }^{19}$. HRs were for the rivaroxaban group as compared with the warfarin group. The RHRs were calculated by dividing simulated HRs by observed HRs for each outcome ${ }^{19}$. Both RDs and RHRs reflect the model error, which could be caused by misspecification of simulation structure or assumptions about input parameters. RDs around 0 and RHRs near to1 represent low error of the simulation model.

\subsection{Comparison of simulated results among ROCKET AF, XANTUS, and two observational studies}

The simulated outcomes for hypothetical cohorts of patients with the marginal covariate distributions similar to XANTUS, and two observational studies were predicted, respectively. Hypothetical cohorts of 7000 rivaroxaban patients were simulated for XANTUS. Similarly, 7000 rivaroxaban patients and 7000 warfarin patients were simulated for the other two observational studies, respectively. The DES model developed and validated in RCT patients was used to estimated outcomes for the three observational study populations. This process was conducted by replacing the baseline characteristics of RCT cohorts with the simulated cohorts of the observational studies. The event rates, RDs, HRs and RHRs were estimated.

\subsection{Statistical analysis}

Demographics and clinical characteristics at baseline for cohorts of involved studies and simulated cohorts were summarized descriptively as means $\pm \mathrm{SD}$ or proportions as appropriate. Key summary measures of DES model were reported as event rates (events per 100 patient-years), RDs, HRs, and RHRs, with associated confidence intervals $\left(95 \%\right.$ CIs) estimated by using the $2.5^{\text {th }}$ and $97.5^{\text {th }}$ percentiles. Simulation of ROCKET AF was repeated 10,20,50,100,200,300, 500, 1000 and 2000 times, respectively, to obtain the optimal iteration times for stable results. Finally, each simulation was repeated 1000 times with the computer running 
time of about 30 minutes. Analyses were performed using STATA software (version13, StataCorp, College Station, Texas, USA).

Results

3.1 Baseline characteristics of observed and simulated patients

Table 1 and Table S3 outline the baseline characteristics of observed and simulated patients in the four studies. The average age of these four studies were all above 70, with the highest mean age of 71.95 in ROCKET AF and the lowest mean age of 71.5 in XANTUS. The proportion of each comorbidity varied from different studies. A larger proportion of $54.9 \%$ rivaroxaban patients with prior stroke/TIA were included in ROCKET AF compared with the three observational studies. Meanwhile, the proportion of patients with heart failure (HF), hypertension, or diabetes mellitus (DM) was also higher in ROCKET AF. Therefore, the mean $\mathrm{CHADS}_{2}$ score was $3.48 \pm 0.94$ in ROCKET AF, which was much higher than that in the three observational studies, indicating the higher stroke risk of patients in ROCKET AF.

\subsection{Simulation of ROCKET AF}

The discrete event simulation model (Figure 1) successfully replicated the overall results of ROCKET AF (Table 2). The simulation was repeated 1000 times to obtain robust and convergent results (Figure S1 and Figure S2). The simulated incidence of stroke/SE and major bleeding was 1.718 vs 1.980 , and 3.463 vs 3.379 per 100 patient-years, for rivaroxaban and warfarin, respectively. The RDs between simulated and observed results were relatively low among each outcome (Table 2). The simulated HRs comparing rivaroxaban and warfarin in the risks of stroke/SE and major bleeding were 0.868 (95\% CI, 0.863-0.872) and 1.025 (95\%CI, 1.021-1.029), respectively (Table 3). The RHRs between simulated and observed results were around 1 . The results indicated that estimated risks and HRs were closely matched the observed risks and HRs in ROCKET $\mathrm{AF}$, representing the low error of the simulation model.

3.3 Counterfactual results of ROCKET AF using the populations in observational studies

Cohorts of equal size and with similar covariate distributions were generated in the XANTUS, Laliberte (2014) and Amin (2017), respectively (Table 1). The baseline characteristics of ROCKET AF were replaced with those of simulated cohorts to repeat the simulation, respectively. The predicted outcomes are shown on Table 3, Table 4, and Table S3-S5. The predicted rates of stroke/SE were 1.718, 1.118, 1.097, and 1.318 per 100 patient-years, while the predicted rates of major bleeding were $3.463,2.817,2.804$, and 3.238 per 100 patient-years in rivaroxaban arms for simulated ROCKET AF, XANTUS, Laliberte (2014) and Amin (2017), respectively. Both predicted rates of stroke/SE and major bleeding were lower in the three observational studies than those in the simulated ROCKET AF (Table 4), with RDs being 0.22-0.66 per 100 patient-years. Whereas, RDs of stroke/SE and major bleeding were similar among the predicted outcomes of the three observational studies, ranging from 0.02-0.43 per 100 patient-years (Table S4 and Table S5). Consistent effects were observed in other simulated outcomes such as stroke, ICH, GI bleeding and MI. Considering the HRs between rivaroxaban and warfarin in each outcome, the simulated HRs of stroke/SE were 0.780 (95\% CI, 0.775-0.785) and 0.824 (95\% CI, 0.819-0.829) for Laliberte (2014) and Amin (2017), respectively, which were close to the observed HRs of 0.77 (95\% CI, 0.55-1.09) and 0.72 (95\% CI, 0.63-0.83) (Table 3). The simulated HRs of major bleeding were 0.940 (95\% CI, 0.936-0.944) for Laliberte (2014) and 1.034 (95\% CI, 1.030-1.039) for Amin (2017), which seemed to be relatively lower than the observed HRs of 1.08 (95\% CI, 0.71-1.64) and 1.17 (95\% CI, 1.10-1.26). Even though some difference was detected between the event rates of each studies, most simulated HRs were similar to the corresponding observed HRs, with most RHRs around 1 (Table 3 ).

Discussion

Both RCT of ROCKET AF and observational studies of XANTUS, Laliberte (2014) and Amin (2017) contributed to the clinical evidence for rivaroxaban in stroke prevention of AF patients. However, the effectiveness and safety of rivaroxaban varied among the four studies. In this study, a DES model was proposed to predict the counterfactual outcomes of ROCKET AF that would have it been conducted in broader observational 
study populations. The DES could successfully replicate the overall results of ROCKET AF. Counterfactual results of ROCKET AF using the populations in observational studies showed relatively lower stroke/SE rate and major bleeding rate than those in simulated ROCKET AF. Moreover, most simulated HRs between rivaroxaban arm and warfarin arm were similar to the corresponding observed HRs, indicating the similar benefits of rivaroxaban in AF patients to ROCKET AF.

As an RCT, ROCKET AF is regarded as gold standard in terms of investigating efficacy and safety of rivaroxaban in AF patients. Nevertheless, ROCKET AF was performed in selected AF patients with moderateto-high risk of stroke (CHADS2 score [?]2 and mean score:3.5), resulting in lack of external validity and generalizability ${ }^{20}$. In comparison, real-world studies, such as XANTUS, Laliberte (2014) and Amin (2017), could reflect real-world treatment patterns among diverse populations, and provide outcome estimates in broad patient populations. In fact, the results of observational studies often differ from those of RCTs and also differ from each other. Difference in patient characteristics could be a common barrier leading to the outcome discrepancies across studies, as baseline covariates, such as age, history of stroke, are also risk factors for the studied outcomes. As a result, the treatment effects might be different across different patient populations. Besides, difference in data source, outcome measures, and patient adherence, as well as confounding bias of observational studies, all contribute to the discrepant results. In addition, the follow-up periods were different in XANTUS, Laliberte (2014) and Amin (2017), ranging from 0.5 to 1 year, which were shorter than the follow-up in ROCKET AF of about 2 years. Considering that some rare events could not be detected and difference in low incidence events might not be found during short follow-up period, the absolute event rates and the relative benefits of rivaroxaban might be inaccurate.

In order to estimate the real-world effectiveness and safety of rivaroxaban in AF patients in a relatively accurate way, we used DES method to model the pathways and 2-year outcomes of rivaroxaban anticoagulation in AF patients. Monte Carlo simulation was used to generate the hypothetical cohorts of patients. DES built in this study could keep track of patient-level covariates and account for the changes in patients' stroke and bleeding risk factors over time ${ }^{9}$. Therefore, the stroke and major bleeding risk could be modified as the patient got older age or greater comorbidity burden. Event rates and treatment effects could then be estimated based on predefined relationships between outcomes and risk factors of stroke and bleeding. The baseline characteristics of ROCKET AF patients were generalized to match the baseline of patients treated in routine care, which facilitated the generation of evidence for effectiveness and safety of rivaroxaban in excluded AF populations.

Our results indicated that even the observed outcomes of ROCKET AF, XANTUS, Laliberte (2014) and Amin (2017) differed from each other, the difference became smaller among the corresponding simulated studies. For the study of Laliberte (2014), wide discrepancies were found in the stroke incidence of rivaroxaban arm between observed and simulated results, with observed rate being 4.6 and simulated rate being 1.097 per 100 patient-years. Similar trend was found in the observed and simulated incidence of major bleeding in Amin (2017). The inconsistency might be caused by the inherent limitations of real-world studies, such as short follow-up, unbalanced confounding bias etc. Interestingly, stroke/SE incidence of rivaroxaban group was close among simulated XANTUS, Laliberte (2014) and Amin (2017) (1.118, 1.097, and 1.318 per 100 patient-years, respectively), which was much lower than the data of simulated ROCKET AF (1.718 per 100 patient-years). It is known that patients enrolled in ROCKET AF were of moderate-to-high stroke risk, with mean $\mathrm{CHADS}_{2}$ score being 3.5. In comparison, the baseline characteristics of these three observational studies were similar and could represent the whole AF population. The stroke risk of patients in the three observational studies was much lower than that in ROCKET AF, with mean CHADS $_{2}$ score being 2.0-2.7. Accordingly, the simulated stroke/SE incidence of the three observational studies might reflect the real-world stoke/SE rate in AF patients using rivaroxaban to some extent, so as the other simulated outcomes.

It is worth noting that most observed and simulated HRs between rivaroxaban and warfarin for each outcome were similar in our study, with most RHRs around 1. In terms of HR for stroke/SE comparing rivaroxaban and warfarin, the simulated HRs in Laliberte (2014) and Amin (2017) were 0.780 and 0.824, respectively, which were close to the observed HR of 0.79 in ROCKET AF. These results, to some extent, confirmed that 
rivaroxaban was noninferior or even superior to warfarin for the prevention of stroke/SE in the real-world setting, which were in accordance with the results in two previous meta-analyses reporting that HRs for stroke/SE comparing rivaroxaban and warfarin were 0.75 (95\% CI, 0.64 to 0.85$)$ and 0.83 (95\% CI, 0.73 to 0.94 ) in real-world setting, respectively ${ }^{21,22}$. With respect to the HR for major bleeding, there was no significant between-group difference, with observed HR being 1.04 in ROCKET AF and simulated HR being 1.034 in Amin (2017). The HRs for major bleeding obtained in this study were also similar to those reported in two previous meta-analyses considering real-world studies, with the HRs being 1.02 (95\% CI, 0.95 to 1.10$)$ and 0.99 (95\% CI, 0.91 to 1.07 ), respectively ${ }^{21,22}$. Therefore, even some differences existed in the absolute rates of stroke/SE and major bleeding between the observed and simulated studies, similar data of effectiveness and safety were detected comparing rivaroxaban and warfarin in the anticoagulation of AF patients.

Some limitations inevitably existed in this study. First, the model error could not be neglected, as DES model structure and pathway was built based on a priori knowledge about disease progression and possible outcomes of $\mathrm{AF}$ patients receiving rivaroxaban, which was lack of multivariable outcome prediction model. Second, $\mathrm{CHA}_{2} \mathrm{DS}_{2}-\mathrm{VAS}_{\mathrm{C}}$ score, rather than $\mathrm{CHADS}_{2}$ score, is now recommended in the clinical guidelines for stroke risk assessment of AF patients, as it has advantages of identifying a subset of low-risk AF patients with $\mathrm{CHADS}_{2}$ score of $0-1^{8,23}$. However, in this study, the relationship between baseline characteristics and clinical outcomes were calculated according to the patient's $\mathrm{CHADS}_{2}$ score, as it was the mainstream score for stroke prediction when ROCKET AF was conducted. Third, as ROCKET AF excluded patients with $\mathrm{CHADS}_{2}$ score of 0 to 1 , the incidence of the events used in the simulation model for this subset of patients was based on RE-LY trail, which investigated the efficacy and safety of dabigatran in AF patients. This could also introduce some error to the model. Moreover, individual-level information was not available in our study. Therefore, bootstrapping method, which could preserve covariance structure among baseline characteristics of observational studies and could increase the accuracy of the simulation, could not be used. In addition, covariates and outcomes of observational studies might be unprecise, as the data were not originally recorded for research purpose and some vital information might be missing. All the mentioned factors could lead to the inaccuracy of the simulation model and the predicted outcomes.

Conclusions

In order to estimate the real-world effectiveness and safety of rivaroxaban in AF patients, DES method was used to model the pathways and 2-year outcomes of rivaroxaban anticoagulation in AF patients. The simulated event incidence of observational studies, such as stroke/SE incidence and major bleeding incidence, which was lower than that in simulated ROCKET AF, might reflect the real-world event rate in AF patients. Even some differences existed in the absolute rates of stroke/SE and major bleeding between the observed and simulated studies, the results confirmed similar effectiveness and safety to ROCKET AF comparing rivaroxaban and warfarin in the anticoagulation of $\mathrm{AF}$ patients

Author Contributions: Zhi-Chun Gu and Chi Zhang designed the study. Mang-Mang Pan and Fang-Hong Shi collected and analyzed the data. Wei-Wei Wang was responsible for methodology and software. Chi Zhang wrote the original manuscript. Zheng Li and Long Shen reviewed and edited the manuscript. All authors have read and agreed to the published version of the manuscript.

Funding

This work was supported by the Research Funds of Shanghai Health and Family Planning commission (20184Y0022), WU JIEPING medical foundation (320.6750.2020-04-30) and Clinical Pharmacy Innovation Research Institute of Shanghai Jiao Tong University School of Medicine (CXYJY2019ZD001, CXYJY2019QN004), and Program for Key but Weak Discipline of Shanghai Municipal Commission of Health and Family Planning (2016ZB0304).

Conflicts of Interest

The authors report no conflicts of interest in this work. 


\section{References}

1. Colilla S, Crow A, Petkun W, Singer DE, Simon T, Liu X. Estimates of current and future incidence and prevalence of atrial fibrillation in the U.S. adult population. Am J Cardiol . Oct 15 2013;112(8):1142-7.

2. Lloyd-Jones DM, Wang TJ, Leip EP, et al. Lifetime risk for development of atrial fibrillation: the Framingham Heart Study. Circulation. Aug 31 2004;110(9):1042-6.

3. January CT, Wann LS, Calkins H, et al. 2019 AHA/ACC/HRS Focused Update of the 2014 AHA/ACC/HRS Guideline for the Management of Patients With Atrial Fibrillation: A Report of the American College of Cardiology/American Heart Association Task Force on Clinical Practice Guidelines and the Heart Rhythm Society in Collaboration With the Society of Thoracic Surgeons. Circulation . Jul 9 2019;140(2):e125-e151.

4. Steffel J, Verhamme P, Potpara TS, et al. The 2018 European Heart Rhythm Association Practical Guide on the use of non-vitamin $\mathrm{K}$ antagonist oral anticoagulants in patients with atrial fibrillation.Eur Heart $J$. Apr 21 2018;39(16):1330-1393.

5. Patel MR, Mahaffey KW, Garg J, et al. Rivaroxaban versus warfarin in nonvalvular atrial fibrillation. $N$ Engl J Med. Sep 8 2011;365(10):883-91.

6. Camm AJ, Amarenco P, Haas S, et al. XANTUS: a real-world, prospective, observational study of patients treated with rivaroxaban for stroke prevention in atrial fibrillation. Eur Heart J . Apr 7 2016;37(14):1145-53.

7. Laliberte F, Cloutier M, Nelson WW, et al. Real-world comparative effectiveness and safety of rivaroxaban and warfarin in nonvalvular atrial fibrillation patients. Curr Med Res Opin . Jul 2014;30(7):1317-25.

8. Lip GY. Stroke and bleeding risk assessment in atrial fibrillation: when, how, and why? Eur Heart $J$. Apr 2013;34(14):1041-9.

9. Wang SV, Schneeweiss S, Gagne JJ, et al. Using Real-World Data to Extrapolate Evidence From Randomized Controlled Trials. Clin Pharmacol Ther. May 2019;105(5):1156-1163.

10. Camm AJ, Amarenco P, Haas S, et al. Real-world vs. randomized trial outcomes in similar populations of rivaroxaban-treated patients with non-valvular atrial fibrillation in ROCKET AF and XANTUS.Europace . Mar 1 2019;21(3):421-427.

11. Amin A, Keshishian A, Trocio J, et al. Risk of stroke/systemic embolism, major bleeding and associated costs in non-valvular atrial fibrillation patients who initiated apixaban, dabigatran or rivaroxaban compared with warfarin in the United States Medicare population.Curr Med Res Opin . Sep 2017;33(9):1595-1604.

12. Blonde L, Khunti K, Harris SB, Meizinger C, Skolnik NS. Interpretation and Impact of Real-World Clinical Data for the Practicing Clinician. Adv Ther . Nov 2018;35(11):1763-1774.

13. Kim H, Kim H, Cho SK, Kim JB, Joung B, Kim C. Cost-Effectiveness of Rivaroxaban Compared to Warfarin for Stroke Prevention in Atrial Fibrillation. Korean Circ J . Mar 2019;49(3):252-263.

14. Oldgren J, Alings M, Darius H, et al. Risks for stroke, bleeding, and death in patients with atrial fibrillation receiving dabigatran or warfarin in relation to the CHADS2 score: a subgroup analysis of the RE-LY trial. Ann Intern Med . Nov 15 2011;155(10):660-7, W204.

15. Goodman SG, Wojdyla DM, Piccini JP, et al. Factors associated with major bleeding events: insights from the ROCKET AF trial (rivaroxaban once-daily oral direct factor Xa inhibition compared with vitamin $\mathrm{K}$ antagonism for prevention of stroke and embolism trial in atrial fibrillation). J Am Coll Cardiol . Mar 11 2014;63(9):891-900. 
16. Mahaffey KW, Stevens SR, White HD, et al. Ischaemic cardiac outcomes in patients with atrial fibrillation treated with vitamin $\mathrm{K}$ antagonism or factor Xa inhibition: results from the ROCKET AF trial. Eur Heart J . Jan 2014;35(4):233-41.

17. Hankey GJ, Stevens SR, Piccini JP, et al. Intracranial hemorrhage among patients with atrial fibrillation anticoagulated with warfarin or rivaroxaban: the rivaroxaban once daily, oral, direct factor Xa inhibition compared with vitamin K antagonism for prevention of stroke and embolism trial in atrial fibrillation. Stroke . May 2014;45(5):1304-12.

18. Sherwood MW, Nessel CC, Hellkamp AS, et al. Gastrointestinal Bleeding in Patients With Atrial Fibrillation Treated With Rivaroxaban or Warfarin: ROCKET AF Trial. J Am Coll Cardiol . Dec 1 2015;66(21):2271-2281.

19. Andrade C. Understanding relative risk, odds ratio, and related terms: as simple as it can get. J Clin Psychiatry . Jul 2015;76(7):e857-61.

20. Camm AJ, Coleman CI, Larsen TB, Nielsen PB, Tamayo CS. Understanding the Value of Real-World Evidence: Focus on Stroke Prevention in Atrial Fibrillation with Rivaroxaban. Thromb Haemost . May 2018;118(S 01):S45-S60.

21. Escobar C, Marti-Almor J, Perez Cabeza A, Martinez-Zapata MJ. Direct Oral Anticoagulants Versus Vitamin K Antagonists in Real-life Patients With Atrial Fibrillation. A Systematic Review and Metaanalysis.Rev Esp Cardiol (Engl Ed) . Apr 2019;72(4):305-316.

22. Bai Y, Deng H, Shantsila A, Lip GY. Rivaroxaban Versus Dabigatran or Warfarin in Real-World Studies of Stroke Prevention in Atrial Fibrillation: Systematic Review and Meta-Analysis. Stroke . Apr 2017;48(4):970-976.

23. Chen JY, Zhang AD, Lu HY, Guo J, Wang FF, Li ZC. CHADS2 versus CHA2DS2-VASc score in assessing the stroke and thromboembolism risk stratification in patients with atrial fibrillation: a systematic review and meta-analysis. J Geriatr Cardiol . Sep 2013;10(3):258-66.

Figure legend

Figure 1. The structure of discrete event simulation (DES) model. DES model structure was built based on a priori knowledge about disease progression and possible outcomes of AF patients. The model was designed to predict treatment outcomes conditional on patients' baseline characteristics. Patients at different stroke and major bleeding risk would trace different probabilistic pathways in the model based on their treatment assignment (rivaroxaban or warfarin). DES could keep track of patient-level covariates and account for the changes in patients' stroke and bleeding risk factors over time. Therefore, the stroke and major bleeding risk could be modified as the patient got older age or greater comorbidity burden. 


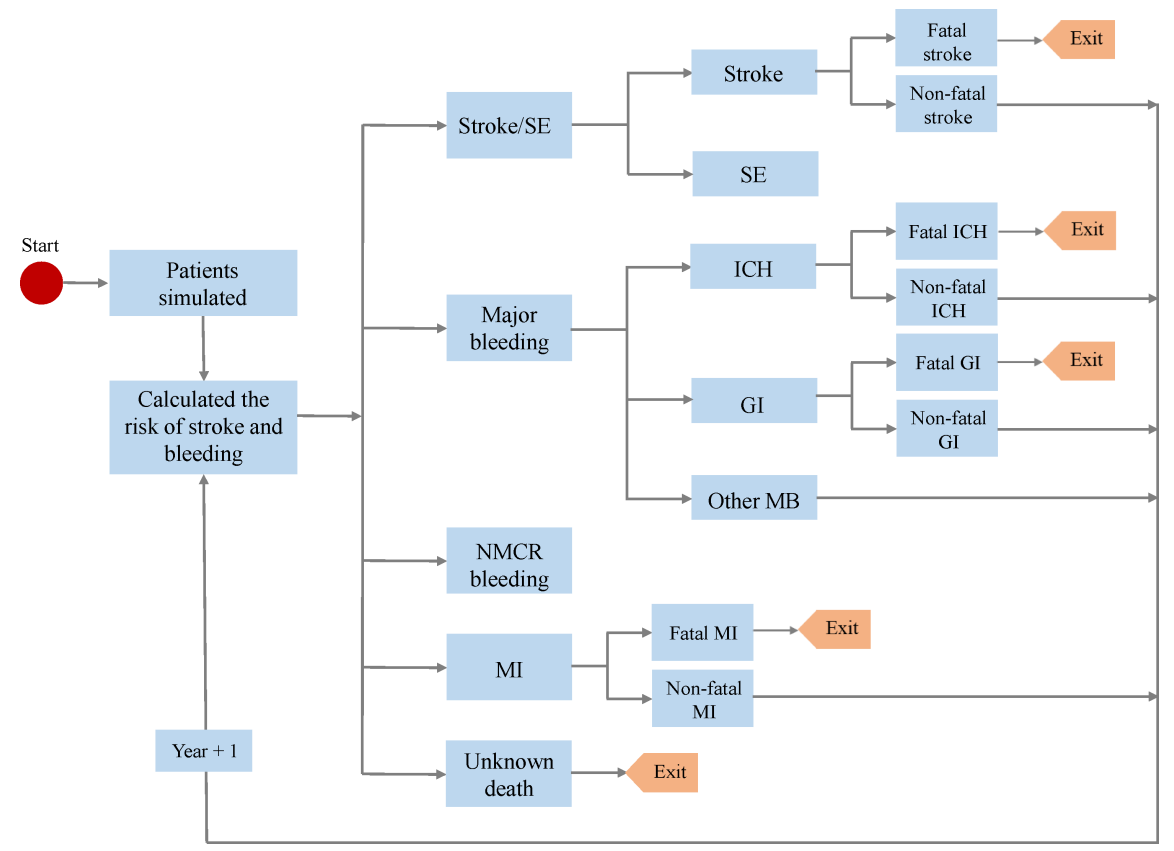

\section{Hosted file}

Table 1.docx available at https://authorea.com/users/350361/articles/475236-simulation-ofanticoagulation-in-atrial-fibrillation-patients-with-rivaroxaban-from-trial-to-targetpopulation

\section{Hosted file}

Table 2.docx available at https://authorea.com/users/350361/articles/475236-simulation-ofanticoagulation-in-atrial-fibrillation-patients-with-rivaroxaban-from-trial-to-targetpopulation

\section{Hosted file}

Table 3.docx available at https://authorea.com/users/350361/articles/475236-simulation-ofanticoagulation-in-atrial-fibrillation-patients-with-rivaroxaban-from-trial-to-targetpopulation

\section{Hosted file}

Table 4.docx available at https://authorea.com/users/350361/articles/475236-simulation-ofanticoagulation-in-atrial-fibrillation-patients-with-rivaroxaban-from-trial-to-targetpopulation 